\title{
Fractional derivatives in electrical circuit theory - critical remarks
}

\author{
RYSZARD SIKORA \\ Department of Electrical Engineering \\ West Pomeranian University of Technology \\ Sikorskiego 37, 70-313 Szczecin, Poland \\ e-mail:rs@zut.edu.pl \\ (Received: 07.09.2016, revised: 01.11.2016)
}

\begin{abstract}
A number of critical remarks related to the application of fractional derivatives in electrical circuit theory have been presented in this paper. Few cases have been pointed out that refer to observed in selected publications violations of dimensional uniformity of physical equation rules as well as to a potential impact on the Maxwell equations.
\end{abstract}

Key words: dimensional uniformity, physical equations, capacity, super capacitor

\section{Introduction}

One may meaningfully benefit in many fields (also in electrical engineering) from the introduction of new mathematical methods or from the reapplication of already existing ones. A mathematical notation models real phenomena. We often face a problem, how to describe particular phenomenon in a comprehensive and at the same time simple way. On one side new phenomena are surfacing and on the other the already known are being understood better. Newton's physics differs from the Einstein's one. Quantum theory resulted with new formulae. Nevertheless, new equations that model the phenomena shall be always dimensionally uniform. Ampere's and Faraday's laws haven't changed as a result of new scientific findings. The dependence between the voltage at the coil and coupled alternate magnetic flux remains the same for decades. The same applies to Maxwellian distribution laws, although we shall fine-tune them with the Lorentz's transformation for high velocities. The implementation of fractional derivatives shouldn't change this principle. It shouldn't impact basic physical laws, including the Maxwellian distribution laws. The implementation of fractional derivatives shall not contradict the laws of physics [1]. This applies to each mathematical description of any

* At the request of part of the reviewers this article has been considered as a discussion article. Therefore, the Editorial Board of AEE turned to the experts indicated by the people interested in the subject matter, to present their views in papers or messages, which will be published in the subsequent issues of AEE. 
phenomenon. A mathematical model of a process must be dimensionally uniform and comply with the valid laws. Each model shall be confirmed with the relevant experiment(s). Unfortunately, the authors who deal with fractional derivatives sometimes don't follow this rule. It's quite common that dimensional uniformity of equations is not followed or misinterpreted by laws of physics that are in use. The papers [2] and [4] as well as many other may serve as an example here. At the same time, many authors notice the dimensional non-uniformity, as to mention [12], page 145, p. 3, where we find: "an auxiliary parameter is introduced in order to preserve the physical dimensionality of the fractional temporal operator". We may find similar references in other works as well.

Till now, the introduction of fractional derivatives has not affected the content of any wellknown textbooks in electrical circuit theory or magnetic field theory. Mentioned textbooks don't refer to fractional derivatives at all. Only derivatives of natural order are used in these books. Similar applies to electromagnetic field wave propagation theory, wave-guide theory, nondestructive testing, electrical machine theory and many other fields of applied science.

\section{Curie's law}

Curie's law presented with the formula below (quoted "as is" from the paper [9]) was applied in the works [9] and [10] to model current in the capacitor:

$$
i(t)=\frac{U_{0}}{h_{1} t^{n}} 0<n<1, \quad t>0 .
$$

The mentioned formula refers both to real (with losses, where $n \neq 1$ ) and ideal capacitors $(n=1)$. In the case of an ideal capacitor the formula (1) will translate into (2):

$$
i(t)=\frac{U_{0}}{h_{1} t} .
$$

It doesn't matter which power exponent $n$ we take, the formula (1) will not be precisely true. The problem with this formula has been flagged by the authors of the paper [9], who wrote namely: "For instance von Schweidler $[2,3]$ was of the opinion that the Curie current is abnormal and named it accordingly.

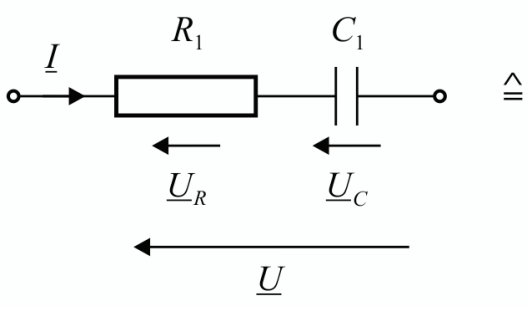

Fig. 1. Serial capacitor scheme

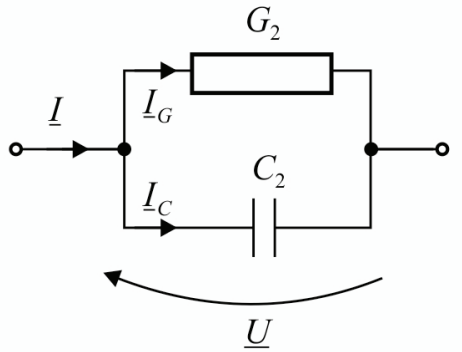

Fig. 2. Parallel capacitor scheme 
Many modern workers assent to the ideas of Schweidler, for instance [4]. But there are also a few who disagree, maybe foremost Jonscher, who in 1977 named the Curie response "the universal dielectric response [5]". Without discussing further the paper [9] we can state that the experimental Curie formula is not the universal one. It is widely used that a real capacitor may be modelled with the dual schemes, serial or parallel [13]. These very simple schemes have been presented in Figs. 1 and 2.

In order to obtain a general model of the capacitor we shall apply one of the canonical schemes (Figs. 3 and 4)

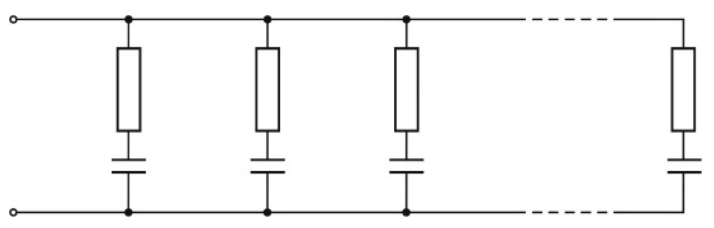

Fig. 3. First canonical scheme

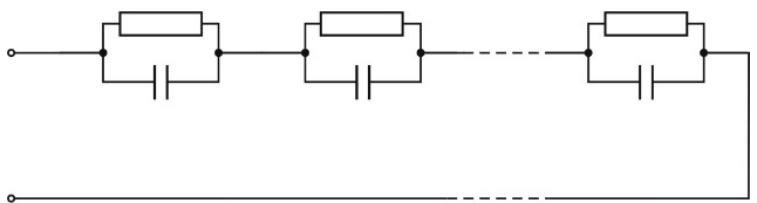

Fig. 4. Second canonical scheme

or Cauer's schemes (Figs. 5 and 6) [13].

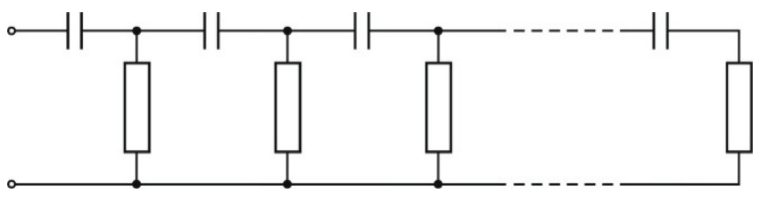

Fig. 5. First Cauer's scheme

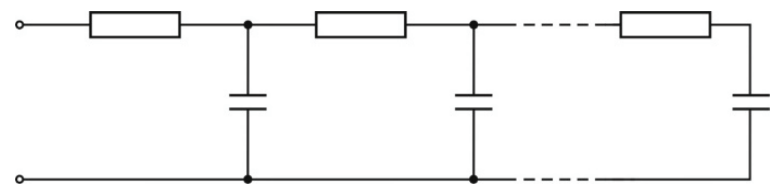

Fig. 6. Second Cauer's scheme

The schemes used may be partial ones. It seems that the scheme presented in Fig. 7 might be feasible.

Resistances $R_{1}$ and $R_{2}$ take finite values in the case of real capacitor $R_{1}=\infty$ and $R_{2}=0$ and for an ideal capacitor. A more general model is represented by one of the long-distance line schemes shown above. 


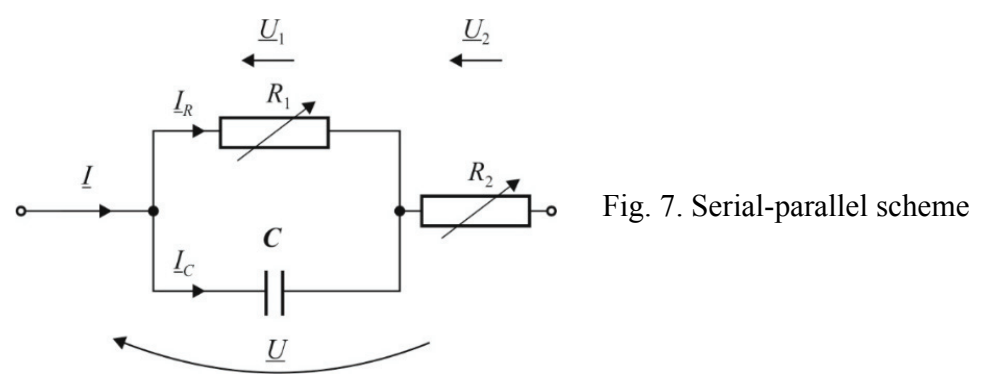

In such a case we don't need to use fractional derivatives that contradict well-established electrical circuit theory, consider how to obtain dimensionally uniform physical equations or reflect whether the dependence between the capacitor's current and voltage shall be described by the well-known formula:

$$
i_{c}(t)=C \frac{\mathrm{d} u_{C}(t)}{\mathrm{d} t}
$$

or by dimensionally non-uniform and physically questionable one:

$$
i_{c}(t)=C \frac{\mathrm{d}^{\alpha} u_{C}(t)}{\mathrm{d} t^{\alpha}}
$$

Dimensional uniformity of physical equation is fulfilled by the formula (3). On the left side we have current, which may be described as coulomb to second. On the right side we find farad multiplied by volt and divided by second, what brings us also to coulomb to second in the end. So we face no doubts.

The problem arises in the case of Eq. (4). As in the previous case we have coulomb to second on the left side, while coulomb to second to the power of $\alpha$ on the right side. And everything works well for $\alpha=1$, but for the case considered by the author of the papers [2, 4] and few other works, when $\alpha \neq 1$ dimensional uniformity is not in place and further discussions aren't correct.

\section{Fractional derivatives in electrical circuit theory}

Many works have been published recently that refer to fractional derivatives, namely [2, 4-9, 10, 14-16]. They play a significant role in automatics and robotics. They may be also useful in other disciplines, e.g. in electrical engineering. In my opinion, the very interesting, fundamental work of prof. T. Kaczorek "Standard and Positive Electrical Circuits with Zero Transfer Matrices" presented at ZKWE'16 and published in Poznan University of Technology Academic Journals Issue 85 2016, which refers to this topic, contains a serious mistake in its crucial part $[2,4]$. Unfortunately, a similar problem appears in many other works that discuss frac- 
tional derivatives. Namely, Eqs. (43-45) and (46) which we can find in the paper [2] and which are presented below as (5), (6), (7) and (8):

$$
\begin{gathered}
i_{c}(t)=\frac{\mathrm{d}^{\alpha} q(t)}{\mathrm{d} t^{\alpha}}, \\
i_{c}(t)=C \frac{\mathrm{d}^{\alpha} u_{C}(t)}{\mathrm{d} t^{\alpha}}, \\
u_{L}(t)=\frac{\mathrm{d}^{\alpha} \Psi(t)}{\mathrm{d} t^{\alpha}}, \\
u_{L}(t)=L \frac{\mathrm{d}^{\alpha} i_{L}(t)}{\mathrm{d} t^{\alpha}},
\end{gathered}
$$

are simply wrong. If to write them correctly, they look as below:

$$
\begin{gathered}
i_{c}(t)=\frac{\mathrm{d} q(t)}{\mathrm{d} t}, \\
i_{c}(t)=C \frac{\mathrm{d} u_{C}(t)}{\mathrm{d} t}, \\
u_{L}(t)=\frac{\mathrm{d} \Psi(t)}{\mathrm{d} t}, \\
u_{L}(t)=L \frac{\mathrm{d} i_{L}(t)}{\mathrm{d} t} .
\end{gathered}
$$

Eqs. (9-11) and (12) have been known for decades. They sometimes appear in the same form as in the works $[2,4]$, but they are complemented with the factor providing dimensional uniformity in such a case. The Faraday's law as presented with the formula (11) has been known for over 100 years. It appears in this form in handbooks, publications and monographs. It hasn't been stated that the mentioned law was applied in the form proposed in the work [2] in any handbook. E.g., Eqs. (6) and (8) could have fulfilled the dimensional uniformity rule, if capacity and inductivity had been functions of time and not measured in farads or henrys.

Consequently, following equations where fractional derivatives were used are wrong as well. If the equations above had been true, the dimensional uniformity rule wouldn't be valid anymore. The discussed equations don't fulfill the mentioned rule.

Let's look at Eq. (5) first. On the left side we have current that may be expressed with coulomb divided by a second, while on the right side we can see coulomb divided by a second to the power with the exponent different than one. This is not correct. A similar problem concerns remaining equations. They are not true as well, so the whole theory established in $[2,4]$ is not true as well and it doesn't matter, if we want to use it for the explanation of the pheno- 
mena in super or classical capacitors. The dimensional uniformity requirement must be met. Acting modes of circuit elements don't influence this rule.

The impact of using the discussed equations is even more serious. Assuming, the mentioned equations were true, this would have influenced the content of the Maxwell equations [14]. The first two Maxwellian distribution laws [14] that create the base of electromagnetism wouldn't have been valid anymore:

$$
\begin{gathered}
\operatorname{rot} \boldsymbol{H}=\boldsymbol{J}+\frac{\partial \boldsymbol{D}}{\partial t}, \\
\operatorname{rot} \boldsymbol{E}=-\frac{\partial \boldsymbol{B}}{\partial t}, \\
\operatorname{rot} \boldsymbol{H}=\boldsymbol{J}+\frac{\partial^{\alpha} \boldsymbol{D}}{\partial t^{\alpha}}, \\
\operatorname{rot} \boldsymbol{E}=-\frac{\partial^{\alpha} \boldsymbol{B}}{\partial t^{\alpha}} .
\end{gathered}
$$

With such an approach we face well-established bases of electromagnetism and the rules of physical formula formulation changing. If the theory presented in the works $[2,4]$ is true, we have to do with the fundamental discovery in the field of electromagnetism. If otherwise, the works $[2,4]$ contain mistake(s).

We may have similar concerns, when we start looking at the huge energy stored by super capacitors. The application of the new technology increased their capacity in relation to volume significantly. However, the amount of the stored energy is determined not only by the capacitor's capacity, but by voltage as well. We may conclude so based on the following formula:

$$
W_{e}=\frac{C U^{2}}{2} .
$$

The energy stored within the electrical (electrostatic) field is a function of energy's volume density:

$$
W_{e}^{\prime}=\frac{\boldsymbol{E} \cdot \boldsymbol{D}}{2},
$$

and of inter-electrode volume and may be written as follows:

$$
W_{e}=\int_{V} W_{e}^{\prime} \cdot \mathrm{d} V .
$$

The energy density of an electrical field is the function of dielectric strength of inter-electrode dielectric and of its permittivity $\varepsilon$. We may increase the electrodes' surface, but we 
won't be able to overcome the limitations resulting from Eq. (18). The maximal stored energy is limited by the finite permittivity $\varepsilon$ values and by the defined electrical strength of the interelectrode dielectric. If we assume that super capacitors don't work basing on the electrostatic rule of energy storing, then we shall define, in what way the energy is stored in such capacitors and then describe this operating mode with relevant formulae.

Is Eq. (47) from the paper [2] correct?

$$
\frac{\mathrm{d}^{\alpha} x(t)}{\mathrm{d} t^{\alpha}}=A x(t)+B u(t) .
$$

It's hard to conclude, as we don't know the dimensions of particular elements. Nevertheless Eq. (48) [2]:

$$
e=R C_{1} \frac{\mathrm{d}^{\alpha} u_{1}}{\mathrm{~d} t^{\alpha}}+u_{1}
$$

is just wrong. For $\alpha$ not equal to 1 the statement

$$
R C_{1} \frac{\mathrm{d}^{\alpha} u_{1}}{\mathrm{~d} t^{\alpha}}
$$

doesn't represent voltage. The dimensional uniformity has been described briefly in p. 2.4 of [13]. This rule may be sometimes helpful for building formulae that describe various physical phenomena. Independently from the need of obeying the dimensional uniformity, the dependences between voltage and current at particular elements of the electrical circuit are precisely defined by the laws of electrical engineering. Let's consider three passive elements $R, L$ and $C$. For these elements the dependence between current and voltage are clearly defined. For resistance this dependence is described by Ohm's law:

$$
i=\frac{u}{R} \text {. }
$$

Ohm's law has exactly the form as above and not any other, e.g.:

$$
i=\frac{u^{\alpha}}{R^{\alpha}}
$$

The same applies to inductivity, which connects current and voltage with the following equations:

$$
\begin{gathered}
u=L \frac{\mathrm{d} i}{\mathrm{~d} t}, \\
\text { or } i=\frac{1}{L} \int u \mathrm{~d} t \text { and not } u=L \frac{\mathrm{d}^{\alpha} i}{\mathrm{~d} t^{\alpha}} .
\end{gathered}
$$

Similarly for capacity: 


$$
\begin{gathered}
u=\frac{1}{C} \int i \mathrm{~d} t \\
\text { or } i=C \frac{\mathrm{d} u}{\mathrm{~d} t} \text { and not } i=C \frac{\mathrm{d}^{\alpha} u}{\mathrm{~d} t^{\alpha}} .
\end{gathered}
$$

In other words, current in the capacitor equals capacity $\mathrm{C}$ multiplied by derivative of voltage at the capacitor. This expresses the physical law. It hasn't been questioned yet - as well as the dependence between voltage at the coil and the derivative of the flux coupled with the coil.

Special "fractional" $R, L, C$ elements have been introduced in the paper [10]. Consequently, special coefficient, ensuring dimensional uniformity has been incorporated in the equations that describe "fractional circuit".

In the paper [10] the Maxwell equations have been presented in the form of (13) and (14), amended with the continuity laws instead of Eqs. (15) and (16). "Classical Equation" has been benchmarked with "Fractional Equation" there as well and dimensional uniformity has been assured. Many simulations have been included in this work as well.

Calculations have been compared to measurements in the paper [16]. A $R C$ circuit has been investigated there. We may conclude from the curves in Fig. 3 in [16] that the transient voltage that has been calculated from the formula

$$
e=R C \frac{\mathrm{d} u_{C}}{\mathrm{~d} t}+u_{C},
$$

matches the experimental results and the one calculated from the formula (21) for the values $\alpha=0.998,0.997,0.996$ only approaches the measurement curve. Another set of calculations has been presented in Fig. 4 in [16]. In this case the results of formula (21) calculations reflect better the measurement curve. Authors explain this fact with non-linearity of circuit elements. However, they don't explain how to take this non-linearity into account, applying fractional derivatives. This may be however realized in the simpler way without fractional derivatives.

\section{Conclusions}

Implementation of new mathematical methods drives the development of many disciplines, including physics. They simplify the complex form of many formulae. For this reason we shall recognize the works related to fractional derivatives that have been touched in this paper. But on this way one may face various problems related to mathematical reflection of physical phenomena. Let's take the mathematical challenges faced by Albert Einstein [17]. At the begining he was stating that when the mathematicians started to analyze his relativity theory he started to have problems with understanding his theory himself. Later he appreciated the role of mathematics and the work of Herman Minkowski. 
Having this in mind, we shall be conscious that even the newest mathematical methods, used for description of physical phenomena shouldn't contradict the physical laws. Complex methods of mathematical modelling may make the understanding of the described phenomenon challenging, but they shouldn't make it completely blurred. I leave it up to audience to opinion, how it is in this particular case.

Future research will aim to recommend the ways of correct application of fractional derivatives in electrical circuit theory and electromagnetic field theory. The topic of local and global solutions for the equations containing fractional derivatives will be in scope of the planned research. Potentially, new types of filters will be designed based on the results of mentioned re-search.

\section{References}

[1] Sikora R., Fractional derivatives in electrical circuits theory-critical remarks, (in Polish), Electrical Review, no. 10, pp. 274-276 (2016).

[2] Kaczorek T., Standard and Positive Electrical Circuits with Zero Transfer Matrices, Poznan University of Technology Academic Journals, no. 85 (2016).

[3] Kaczorek T., Zeroing of state variables in fractional descriptor electrical circuits by state-feedbacks. Archives of Electrical Engineering, vol. 63(249), no. 3 (2014).

[4] Kaczorek T., Positivity and Reachability of Fractional Electrical Circuits, Acta Mechanica et Automatica, vol. 5, no. 2 (2011).

[5] Domek S., Application of fractional derivatives calculus in predictive control, (in Polish), West Pomeranian University of Technology Publishing, Szczecin (2013).

[6] Domek S., Dworak P., Theoretical Developments and Application of Non-Integer Order Systems, Springer (2016).

[7] Włodarczyk M., Zawadzki A., Positive order fractional derivatives in RLC circuits, (in Polish), Electrics, no. 1(217) (2011).

[8] Zawadzki A., Włodarczyk M., Modelling of super capacitor's charging and discharging processes, (in Polish), Measurements, Automatics, Control, vol. 56, no. 12, pp. 1413-1415 (2010).

[9] Westerlund S., Ekstam L., Capacitor Theory, IEEE Transaction on Dielectric and Electrical Insulation, vol. 1, no. 5, pp. 826-839 (1994).

[10] Morales M.A., Lainez R., Mathematical Modelling of Fractional Order Circuits, arXiv: 1602.03541v1 [physics. class-ph], vol. 21 (2016).

[11] Povstenko Y., Solutions to time-fractional diffusion-wave equation in spherical coordinates, Acta Mechanica et Automatica, vol. 5, no. 2, pp. 108-111 (2011).

[12] Gomez-Aguilar J.F., et al., Electrical circuits described by a fractional derivative with regular Kernel, Revista Mexicana de Fisica, vol. 62 (2016).

[13] Sikora R., Chady T., Łopato P., Psuj G., Theoretical Electrical Engineering, (in Polish), West Pomeranian University of Technology Publishing, Szczecin (2016).

[14] Sikora R., Electromagnetic field theory, (in Polish), WNT Warszawa (1997).

[15] Gomez-Aguilar J.F., Behavior characteristics of a cap-resistor, memcapacitor, and a memristor from the response obtained of $R C$ and $R L$ electrical circuits described by fractional differential equations, Turkish Journal of Electrical Engineering \& Computer Sciences, no. 24, pp. 1421-1433 (2016).

[16] Erti H., Calik A.E., Sirin H., Sen M., Oder B., Investigation of electrical RC circuit within the framework of fractional calculus, Revista Mexicana de Fisica, vol. 61, pp. 58-63 (2015).

[17] Kaku M., Einstein's universe, (in Polish), Pruszynski \& Co (2004). 